\title{
Convective Heat and Mass Transfer Flow of Nanofluid past a Vertical Wavy Wall with Thermal Radiation, Chemical Reaction with Space and Temperature Dependent Heat Sources
}

\author{
Dr. M. Nagasasikala* \\ Lecturer in Mathematics, Government Degree College (A) Anantapuramu, A.P., India.
}

*Corresponding Author: Dr. M. Nagasasikala, Lecturer in Mathematics, Government Degree College (A) Anantapuramu, A.P., India.

\begin{abstract}
Coupled effect of thermal radiation, heat sources and chemical reaction on natural convective heat and mass transfer flow over a vertical wavy surface embedded in a fluid saturated porous medium is considered. The governing equations for flow heat and mass transfer are transformed to a plane geometry case by employing the Rungre-Kutta fourth order with shooting technique. The non-dimensional velocity, temperature and nanoconcentration graphs as well as skin friction, rate of heat and mass transfer coefficients are displayed for different values of radiation parameter, heat source parameter, chemical reaction and amplitude of the wavy surface.
\end{abstract}

Keywords: Nanofluid, Wavy Wall, Thermal Radiation, Chemical reaction, Heat Sources.

\section{INTRODUCTION}

Magnetic nanofluid has both the liquid and magnetic properties. These fluids have been used in magneto-optical wavelength filters, optical gratings, optical switches etc. The study of Hall effects on nanofluid has applications in MHD energy generators. Study on the problem of Hall currents in nanofluids over a stretching sheet is very limited. Several authors Abo-Eldahab and Abd El-Aziz [1], Ali et al. [3]. Abo-Eldahab and Salem [2], Xiaohonh Su and Liancun Zheng [15], Mohamed Abd ElAziz [8]] investigated the effect of Hall currents on convective heat transfer flow of nanofluid past stretching sheet und varied conditions.

In recent years energy and material saving considerations have prompted an expansion of the efforts at producing efficient heat exchanger equipment through augmentation of heat transfer.Hence it is advantageous to go for converging-diverging geometries for improving the design of heat transfer equipment.

Several authors [Rees \& Pop[11,12], Ching-Yang Cheng [4], Hossain et al [6], Hady et al [5], Mallikarjuna [7], Vendabai [14], Ramakrishna and Satyanarayana [9], Ranganatha reddy et al[10]] have discussed heat and mass transfer fluid flow past wavy surface at different condition.

Sulochana et al [13] have analysed MHD flow over a permeable stretching /shrinking sheet of a nanofluid in porous medium with suction/injection along with viscous dissipation, considering nanofluid volume fraction on the boundary is submissive to controlled rather than active controlled.

In this paper, we investigate effect of thermal radiation,heat sources,chemical reaction on natural nondarcy convective heat and mass transfer flow over a vertical wavy surface embedded in a fluid saturated porous medium along with viscous dissipation.Here we considered nanofluid volume fraction on the boundary is submissive controlled rather than active controlled.. The vertical wavy wall and the governing equations for flow heat and mass transfer are transformed to a plane geometry case by employing the Runge-Kutta fourth order with shooting technique. The non-dimensional velocity, temperature and concentration graphs as well as skin friction, rate of heat and mass transfer coefficients are displayed for different values of thermal radiation, heat source, chemical reaction parameter and amplitude of the wavy surface. 


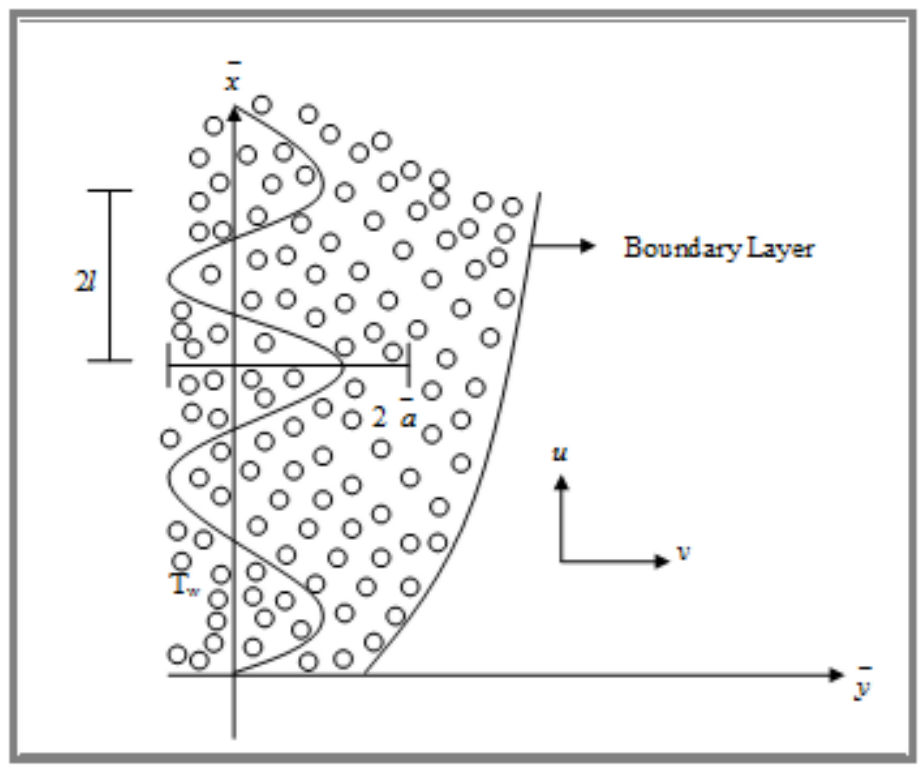

Fig, - 1 : Physical Configuration and Co-ordinate System

\section{Formulation of the Problem}

We analyser steady incompressible two-dimensional laminar natural convective heat and mass transfer flow of a nanofluid over a vertical wavy surface embedded in a saturated porous medium. The porous medium is uniform and local thermal equilibrium with the fluid. The Darcy law is used to describe the fluid saturated porous medium. The fluid is assumed to be gray absorbing-emitting radiation but non-scattering medium.

The wavy surface profile is given by

$y=\bar{\sigma}(\bar{x})=\bar{a} \operatorname{Sin}\left(\frac{\pi \bar{x}}{l}\right)$

where $l$ is the characteristic length of wavy surface and $\bar{a}$ is the amplitude of the wavy surface. The wavy surface is maintained at constant temperature $\mathrm{Tw}$ which are higher than the ambient fluid temperature $T_{\infty}$. We consider the natural convection-radiation flow in the presence of heat sources to be governed by the following equations under Boussinesq approximations:

$$
\begin{aligned}
& \frac{\partial u}{\partial x}+\frac{\partial v}{\partial y}=0 \\
& u=-\frac{k}{\mu} \frac{\partial p}{\partial x}+\frac{k g\left(1-C_{\infty}\right) \beta \rho_{f_{\infty}} \beta g\left(T-T_{\infty}\right)}{\mu}-\frac{\left(\rho_{p}-\rho_{f_{\infty}}\right) k g\left(C-C_{\infty}\right)}{\mu}-\frac{k \sigma \mu_{e}^{2} H_{0}^{2}}{\mu} u-\left(\frac{b}{\sqrt{k}}\right) u^{2} \\
& v=-\frac{k}{\mu} \frac{\partial p}{\partial y}-\left(\frac{b}{\sqrt{k}}\right) v^{2} \\
& u \frac{\partial T}{\partial x}+v \frac{\partial T}{\partial y}=\frac{k_{f}}{\rho_{f} C_{p}} \frac{\partial^{2} T}{\partial y^{2}}+\tau\left\{D_{B}\left(\frac{\partial T}{\partial x} \frac{\partial C}{\partial x}+\frac{\partial T}{\partial y} \frac{\partial C}{\partial y}\right)+\frac{D_{T}}{T_{\infty}}\left(\left(\frac{\partial T}{\partial x}\right)^{2}+\left(\frac{\partial T}{\partial y}\right)^{2}\right)\right]-\left(\frac{1}{\rho C_{p}}\right) \frac{\nabla \cdot q_{R}}{\partial y}+\frac{q^{\prime \prime \prime}}{\rho C_{p}} \\
& u \frac{\partial C}{\partial x}+v \frac{\partial C}{\partial y}=D_{B}\left(\frac{\partial^{2} C}{\partial x^{2}}+\frac{\partial^{2} C}{\partial y^{2}}\right)+\frac{D_{T}}{T_{\infty}}\left(\frac{\partial^{2} T}{\partial x^{2}}+\frac{\partial^{2} T}{\partial y^{2}}\right)-k c\left(C-C_{\infty}\right)
\end{aligned}
$$

The relevant boundary conditions are

$$
\vec{u}=0, \bar{v}=0, T=T_{w}, D_{B} \frac{\partial C}{\partial y}+k_{f} \frac{\partial T}{\partial y}=0 \text { at } \bar{y}=\bar{\sigma}(\bar{x})=\bar{a} \operatorname{Sin}\left(\frac{\pi \bar{x}}{l}\right)
$$


$\vec{u}=0, T \rightarrow T_{\infty}, C \rightarrow C_{\infty}$ as $\bar{y} \rightarrow \infty$

Where $\bar{u}$ and $\bar{v}$ are the volume averaged velocity components in the directions of $\mathrm{x}$ and $\mathrm{y}$ respectively T, $\mathrm{C}$ are temperature, Concentration respectively, $\rho$ is the density of the fluid, $\mu$ is the dynamic viscosity of the fluid, $\mathrm{k}$ is the permeability of the porous medium, $\sigma$ is the electrical conductivity, $\mu_{e}$ is the magnetic permeability, Ho is the strength of the magnetic field, $D_{\mathrm{B}}$ is the Brownian diffusion coefficient and $\mathrm{D}_{\mathrm{T}}$ is the thermophoretic diffusion coefficient, $\tau=\frac{\left(\rho C_{p}\right)}{\left(\rho C_{f}\right)}$ is the ratio between the effective heat capacity of the nanoparticle material and heat capacity of the fluid, kc is the coefficient of chemical reaction, $\beta_{o}$ are the coefficients of thermal expansion, $\alpha$ is the thermal conductivity, $q_{r}$ is the radiative heat flux, $\mathrm{g}$ is the acceleration due to gravity, $q^{\prime \prime \prime}$ is rate of internal heat generation $(>0)$ or absorption $(<0)$ coefficient and $\mathrm{Q}_{\mathrm{H}}$ is the strength of the heat source.

Eliminating pressuire from equations (3) \& (4) we get

$\left(1+M^{2}\right) \frac{\partial u}{\partial y}-\frac{\partial v}{\partial x}=\frac{k g\left(1-C_{\infty}\right) \beta \rho_{f_{\infty}} \beta g}{\mu} \frac{\partial T}{\partial y}-\frac{\left.\left(\rho_{p}-\rho_{f_{\infty}}\right) k g\right)}{\mu} \frac{\partial C}{\partial y}-\left(\frac{2 b}{\bar{k}}\right)\left(u \frac{\partial u}{\partial y}-v \frac{\partial v}{\partial x}\right)$

where $M^{2}=\frac{k \sigma \mu_{e}^{2} H_{o}^{2}}{\mu}$ is the magnetic field parameter.

On using Rosseland approximation the energy equation with non-uniform heat sources reduces to

$$
\begin{aligned}
& \rho C_{p}\left(\bar{u} \frac{\partial T}{\partial \bar{x}}+\bar{v} \frac{\partial T}{\partial \bar{y}}\right)=k_{f}\left(\frac{\partial^{2} T}{\partial x^{2}}+\frac{\partial^{2} T}{\partial y^{2}}\right)+\frac{16 \sigma^{\bullet} T_{\infty}^{3}}{3 \beta_{R}}\left(\frac{\partial^{2} T}{\partial x^{2}}+\frac{\partial^{2} T}{\partial y^{2}}\right)+ \\
& +\frac{k R a}{L^{2} \xi}\left[A^{\bullet}\left(T_{w}-T_{\infty}\right) f^{\prime}+B^{\bullet}\left(T-T_{\infty}\right)\right] / \rho C_{p}+\frac{D_{T}}{T_{\infty}}\left(\left(\frac{\partial T}{\partial x}\right)^{2}+\left(\frac{\partial T}{\partial y}\right)^{2}\right)
\end{aligned}
$$

On introducing the following non-dimensional variables

$$
x=\frac{\bar{x}}{l}, y=\frac{\bar{y}}{l}, a=\frac{\bar{a}}{l}, \sigma=\frac{\bar{\sigma}}{l}, \psi^{*}=\frac{\bar{\psi}}{l}, \theta=\frac{T-T_{\infty}}{T_{w}-T_{\infty}}, \phi=\frac{C-C_{\infty}}{C_{w}-C_{\infty}}, \bar{u}=\frac{\partial \bar{\psi}}{\partial \bar{y}} \quad, v=-\frac{\partial \bar{\psi}}{\partial \bar{x}}
$$

Equations (6),(8) \& (9) reduce to

$$
\begin{aligned}
& \frac{\partial^{2} \psi^{\bullet}}{\partial x^{2}}+\left(1+M^{2}\right) \frac{\partial^{2} \psi^{\bullet}}{\partial y^{2}}=R a\left(\frac{\partial \theta}{\partial y}-N \frac{\partial \phi}{\partial y}\right)-f_{S} D^{-1}\left(\frac{\partial \psi^{\bullet}}{\partial y} \frac{\partial^{2} \psi^{\bullet}}{\partial y^{2}}-\frac{\partial \psi^{\bullet}}{\partial x} \frac{\partial^{2} \psi^{\bullet}}{\partial x^{2}}\right) \\
& \left(1+\frac{4 R d}{3}\right)\left(\frac{\partial^{2} \theta}{\partial \vec{x}^{2}}+\frac{\partial^{2} \theta}{\partial \bar{y}^{2}}\right)+N b\left(\frac{\partial \theta}{\partial \bar{x}} \frac{\partial \phi^{\bullet}}{\partial \bar{y}}+\frac{\partial \theta}{\partial \bar{y}} \frac{\partial \phi^{\bullet}}{\partial \bar{x}}\right)+N t\left(\left(\frac{\partial \theta}{\partial \bar{x}}\right)^{2}+\left(\frac{\partial \theta}{\partial y}\right)^{2}\right)+ \\
& +A_{11} f^{\prime}+B_{11} \theta=\left(\frac{\partial \theta}{\partial x} \frac{\partial \psi^{\bullet}}{\partial y}-\frac{\partial \theta}{\partial y} \frac{\partial \psi^{\bullet}}{\partial x}\right) \\
& \frac{1}{L e}\left(\frac{\partial^{2} \phi}{\partial \bar{x}^{2}}+\frac{\partial^{2} \phi}{\partial \bar{y}^{2}}\right)+\left(\frac{N t}{N b}\right)\left(\frac{\partial^{2} \phi}{\partial \bar{x}^{2}}+\frac{\partial^{2} \phi}{\partial \bar{y}^{2}}\right)-\operatorname{Le\gamma \phi }=-\left(\frac{\partial \phi}{\partial \bar{x}} \frac{\partial \psi^{\bullet}}{\partial \bar{y}}-\frac{\partial \phi}{\partial \bar{y}} \frac{\partial \psi^{\bullet}}{\partial \bar{x}}\right)
\end{aligned}
$$

where $\quad R a=\frac{L g \beta\left(T_{w}-T_{\infty}\right)\left(1-C_{\infty}\right)}{a \mu}, \quad v=\frac{\mu_{\infty}}{\rho}, \quad R d=\frac{4 \sigma^{*} T_{\infty}^{3}}{k_{f} \beta_{R}}, \quad M^{2}=\frac{\sigma \mu_{e}^{2} H_{0}^{2} l^{2}}{\mu}, L e=\frac{v}{D_{B}}$, $\gamma=\frac{k c L^{2}}{D_{B}}, N=\frac{\left(\rho_{p}-\rho_{f \infty}\right)\left(C_{w}-C_{\infty}\right)}{\rho_{f \infty}\left(1-C_{\infty}\right)\left(T_{w}-T_{\infty}\right)}, N_{b}=\frac{\tau D_{B}\left(C_{w}-C_{\infty}\right)}{a}, N_{t}=\frac{\tau D_{T}\left(T_{w}-T_{\infty}\right)}{a T_{\infty}}, f_{s}=\frac{2 b}{\mu l}$ 
is the Darcy-Rayleigh number, kinematic viscosity of the fluid, Radiation parameter, magnetic parameter, Lewis number, chemical reaction parameter, buoyancy ratio, Brownian motion parameter, Thermophoresis parameter, the Forchheimer parameter.

The transformed boundary conditions are

$$
\begin{aligned}
& \psi^{\bullet}=0, \theta=1, N b \frac{\partial \phi}{\partial y}+N t \frac{\partial \theta}{\partial y}=0 \text { at } y=a \operatorname{Sin}(x) \\
& \frac{\partial \psi^{\bullet}}{\partial y} \rightarrow 0, \theta \rightarrow 0, \phi \rightarrow \infty \text { as } y \rightarrow \infty
\end{aligned}
$$

We can transform the effect of wavy surface from the boundary conditions into the governing equations by using suitable coordinate transformation with boundary layer scaling for the case of free convection. The Cartesian coordinates (x, y) are transformed into the new variables $(\xi \eta)$.

We incorporate the effect of effect of wavy surface and the usual boundary layer scaling into the governing equations (11)-(13) for free convection using the transformations and $R a \rightarrow \infty$ (i.e boundary layer approximation)

$$
x=\xi, \bar{\eta}=\frac{y-a \operatorname{Sin}(x)}{\xi^{1 / 2} R a^{-1 / 2}}, \psi^{*}=R a^{1 / 2} \psi
$$

These transformations are similar to those presented in for instance Rees and Pop [12]. We obtain the following boundary layer equations :

$$
\begin{gathered}
\begin{array}{c}
\left(1+a^{2} \operatorname{Cos}^{2} \xi\right) \frac{\partial \theta}{\partial \eta} \frac{\partial \psi}{\partial \eta}+\left(1+a^{2} \operatorname{Cos}^{2} \xi\right) \frac{\partial^{2} \psi}{\partial \eta^{2}}=R a \xi^{1 / 2}\left(\frac{\partial \theta}{\partial \eta}-N \frac{\partial \phi}{\partial \eta}\right)-M^{2} \frac{\partial^{2} \psi}{\partial \eta^{2}} \\
-f_{s} D^{-1}\left(1+a^{3} \operatorname{Cos}^{3}(\xi)\right) \frac{\partial \psi}{\partial \eta} \frac{\partial^{2} \psi}{\partial \eta^{2}}
\end{array} \\
\begin{array}{c}
\xi^{1 / 2}\left(\frac{\partial \theta}{\partial \xi} \frac{\partial \psi}{\partial \eta}-\frac{\partial \theta}{\partial \eta} \frac{\partial \psi}{\partial \xi}\right)=\left(1+a^{2} \operatorname{Cos}^{2}(\xi)\right)\left(\left(1+\frac{4 R d}{3}\right)\left(\frac{\partial^{2} \theta}{\partial \eta^{2}}\right)+\mid\right. \\
+N b \frac{\partial \theta}{\partial \eta} \frac{\partial \phi}{\partial \eta}+N t\left(\frac{\partial \theta}{\partial \eta}\right)^{2}+A_{11} f^{\prime}+B_{11} \theta
\end{array} \\
\xi^{1 / 2} \operatorname{Le}\left(\frac{\partial \phi}{\partial \xi} \frac{\partial \psi}{\partial \eta}-\frac{\partial \phi}{\partial \eta} \frac{\partial \psi}{\partial \xi}\right)=\left(1+a^{2} \operatorname{Cos}^{2}(\xi)\right)\left(\frac{\partial^{2} \phi}{\partial \eta^{2}}+\frac{N t}{N b} \frac{\partial^{2} \phi}{\partial \eta^{2}}\right)-\operatorname{Le\gamma }(\phi)
\end{gathered}
$$

\section{Soulution Methodology}

We now introduce the following similarity variables as

$$
\eta=\frac{\bar{\eta}}{\left(1+a^{2} \operatorname{Cos}^{2}(\xi)\right)}, \psi=\xi^{1 / 2} f(\eta), \theta=\theta(\eta), \phi=\phi(\eta)
$$

In equations (15)-(17)we obtain a system of ordinary differential equations as follows:

$$
\begin{aligned}
& f^{\prime \prime}+\theta^{\prime} f^{\prime}-\frac{M^{2}}{\left(1+a^{2} \operatorname{Cos}^{2}(\xi)\right)} f^{\prime \prime}=\operatorname{Ra}\left(\theta^{\prime}-N r \phi^{\prime}\right)-f s d^{-1}\left(\frac{1+a^{3} \operatorname{Cos}^{3}(\xi)}{\left(1+a^{2} \operatorname{Cos}^{2}(\xi)^{2}\right)}\right) f^{\prime \prime} f^{\prime \prime} \\
& \left(1+\frac{4 R d}{3}\right) \theta^{\prime \prime}+\frac{1}{2} f \theta^{\prime}+N b \theta^{\prime} \phi^{\prime}+N t\left(\theta^{\prime}\right)^{2}+\frac{A_{11} f^{\prime}+B_{11} \theta}{\left(1+a^{2} \operatorname{Cos}^{2}(\xi)\right)} \\
& \phi^{\prime \prime}+\frac{L e}{2} f \phi^{\prime}+\left(\frac{N t}{N b}\right) \theta^{\prime \prime}-\operatorname{Le\gamma }\left(1+a^{2} \operatorname{Cos}^{2}(\xi)\right) \phi
\end{aligned}
$$


Where prime denotes differentiation with respect to $\eta$.

The corresponding boundary conditions are

$f=0 \theta=1 \quad N b \frac{\partial \phi}{\partial y}+N t \frac{\partial \theta}{\partial y}=0$ at $\eta=0$

$f^{\prime} \rightarrow 0 \theta \rightarrow 0 \phi \rightarrow 0$ as $\eta \rightarrow \infty$

The main results of practical interest in many applications are heat transfer coefficient mass transfer coefficient at the surface. The drag,heat and mass transfer coefficients are expressed in terms of skin friction, Nusselt and Sherwood numbers $\mathrm{C}_{\mathrm{f}}$, Nux, Shx. Skin friction, $\left(\mathrm{C}_{\mathrm{f}}\right)$, Nusselt number (Nux) and Sherwood number (Shx) are defined in terms of Rax and the amplitude ' $a$ ' as

$$
N u x=\frac{x q_{w}}{\alpha_{o}\left(T_{w}-T_{\infty}\right)}, \quad S h x=\frac{x m_{w}}{D_{b}\left(C_{w}-C_{\infty}\right)}
$$

where $\mathrm{q}_{\mathrm{w}}$ is the heat flux on the wavy surface and is defined by

$q_{w}=-\alpha_{0} \bar{n} . \nabla T$ and $\bar{n}=\left(-\frac{a \operatorname{Cos}(\xi)}{\sqrt{\left(1+a^{2} \operatorname{Cos}^{2}(\xi)\right)}}, \frac{1}{\sqrt{\left(1+a^{2} \operatorname{Cos}^{2}(\xi)\right)}}\right)$ is the unit normal vector to the wavy surface $\alpha_{0}$ is the effective porous medium thermal conductivity.

Therefore

$$
C_{f}=\frac{f^{\prime \prime}(0)\left(1+a^{2} \operatorname{Cos}^{2}(\xi)\right) R a_{x}^{1 / 2}}{\left(1+M^{2}+a^{2} \operatorname{Cos}^{2}(\xi)\right)}, N u_{\xi}=-\frac{\theta^{\prime}(0) R a_{\xi}^{1 / 2}}{\sqrt{\left(1+a^{2} \operatorname{Cos}^{2}(\xi)\right)}}, S h_{\xi}=-\frac{\phi^{\prime}(0) R a_{x}{ }^{1 / 2}}{\left(1+a^{2} \operatorname{Cos}^{2}(\xi)\right)}
$$

\section{CONPARISON}

In the absence of magnetic field $(\mathrm{M}=0)$ and heat sources $(\mathrm{A} 11=0 \& \mathrm{~B} 11=0)$, temperature ratio $(\mathrm{A}=\theta \mathrm{w}=1)$ and chemical reaction $(\gamma=0)$ the results are in good agreement with Vendabai et al [14]

\begin{tabular}{|c|c|c|c|c|c|}
\hline \multirow{2}{*}{\multicolumn{2}{|c|}{ Parameter }} & \multicolumn{2}{|c|}{ Vendabai et al [14] } & \multicolumn{2}{|c|}{ Present Results } \\
\hline & & $\tau(\mathbf{0})$ & Nu(0) & $\tau(\mathbf{0})$ & $\mathbf{N u ( 0 )}$ \\
\hline \multirow{4}{*}{$\mathrm{Ra}$} & 2 & -0.384567 & 0.552361 & -0.384557 & 0.552357 \\
\hline & 4 & -0.413678 & 0.507823 & -0.413665 & 0.507817 \\
\hline & 6 & -0.537896 & 0.205912 & -0.537888 & 0.205909 \\
\hline & 10 & -0.608976 & 0.196485 & -0.608973 & 0.196465 \\
\hline \multirow{4}{*}{ A1 } & -0.2 & -0.365436 & 0.632326 & -0.365432 & 0.632320 \\
\hline & -0.4 & -0.467885 & 0.652293 & -0.467880 & 0.652288 \\
\hline & 0.2 & -0.105678 & 0.400191 & -0.105674 & 0.400187 \\
\hline & 0.4 & -0.135679 & 0.206583 & -0.135672 & 0.206578 \\
\hline \multirow{4}{*}{ B1 } & -0.2 & -0.345678 & 0.552361 & -0.345675 & 0.552351 \\
\hline & -0.4 & -0.554463 & 0.806242 & -0.554453 & 0.806238 \\
\hline & 0.2 & 0.300211 & 0.109467 & 0.300208 & 0.109461 \\
\hline & 0.4 & 0.167877 & 0.334523 & 0.167872 & 0.334517 \\
\hline \multirow{4}{*}{$\mathrm{Nb}$} & 0.1 & -0.505678 & 0.552361 & -0.505675 & 0.552357 \\
\hline & 0.3 & -0.323453 & 0.632362 & -0.323452 & 0.632358 \\
\hline & 0.4 & -0.267898 & 0.607824 & -0.267888 & 0.607818 \\
\hline & 0.5 & -0.203885 & 0.534872 & -0.203880 & 0.534865 \\
\hline \multirow{4}{*}{$\mathrm{Nt}$} & 0.1 & -0.299288 & 0.552361 & -0.299287 & 0.552357 \\
\hline & 0.2 & -0.324728 & 0.452362 & -0.324724 & 0.452358 \\
\hline & 0.3 & -0.367809 & 0.436086 & -0.367802 & 0.436081 \\
\hline & 0.4 & -0.399432 & 0.352556 & -0.399427 & 0.352552 \\
\hline
\end{tabular}

Table1. Comparison 
Convective Heat and Mass Transfer Flow of Nanofluid past a Vertical Wavy Wall with Thermal Radiation, Chemical Reaction with Space and Temperature Dependent Heat Sources

\begin{tabular}{|c|r|r|r|r|r|}
\hline \multirow{4}{*}{$\mathrm{a}$} & 0.1 & -0.337865 & 0.552361 & -0.337845 & 0.552359 \\
\cline { 2 - 6 } & 0.2 & -0.321345 & 0.468091 & -0.321340 & 0.468088 \\
\cline { 2 - 6 } & 0.3 & -0.310678 & 0.371734 & -0.310675 & 0.371726 \\
\cline { 2 - 6 } & 0.4 & -0.301151 & 0.282947 & -0.301147 & 0.282936 \\
\hline \multirow{3}{*}{$\xi$} & $\pi / 6$ & -0.308474 & 0.552361 & -0.308470 & 0.552354 \\
\cline { 2 - 6 } & $\pi / 4$ & -0.299728 & 0.552456 & -0.299724 & 0.552449 \\
\cline { 2 - 6 } & $\pi / 3$ & -0.298475 & 0.552567 & -0.298465 & 0.552558 \\
\cline { 2 - 6 } & $\pi / 2$ & -0.299466 & 0.552667 & -0.299461 & 0.552657 \\
\hline
\end{tabular}

\section{RESULTS AND DISCUSSION}

We follow the convention that the non-dimensional concentration is positive/negative according as the actual concentration $(\mathrm{C})$ is greater/lesser than the ambient concentration $(\mathrm{C} \infty)$. While the temperature is positive for all variations.

Figs.2a-2c depicts the variation of velocity, temperature and nano-concentration with buoyancy ratio $(\mathrm{N})$. It can be seen from the profiles that when the molecular buoyancy force dominates over the thermal buoyancy force the velocity enhances in the region $(0,1)$ and reduces in the region $(1,4)$ when the buoyancy forces are in the same direction (fig.2a). The temperature enhances with increase in $\mathrm{N}$ (fig.2b).Also the actual nanoconcentration enhances in the region $(0,1.0)$ and reduces in the region $(1.0,4.0)$ with increasing $\mathrm{N}$ (fig.2c).

Figs.3a-3c show the variation of velocity, temperature and nano-concentration with the influence of radiation parameter $(\mathrm{Rd})$. From fig.3a we find that the velocity reduces in the region $(0,0.5)$ and enhances in the region $(0.5,4.0)$. This means that the thickness of the momentum boundary layer reduces with increasing values of Rd. Fig. $3 b$ represent the temperature with Rd. It can be seen from the profiles that an increase in Rd leads to thickening of the thermal boundary layer.The actual nanoconcentration enhances ion the flow region $(0,0.5)$ and reduces in the remaining region(0.5,4.0).(fig.3c).

Figs.4a-4c represent $\mathrm{u}, \theta$ and $\phi$ with Eckert number $(\mathrm{Ec})$. From the profiles we notice that higher the dissipation smaller the velocity, larger the temperature and the nano-concentration in the flow region.

Figs.5a-5c and \& $6 a-6 c$ represent $u, \theta$ and $\phi$ with space dependent heat source parameter (A1) and temperature dependent heat source parameter (B1). From fig.5a \& 6a we find that in the region $(0$, $0.2)$ the velocity reduces with $\mathrm{A} 1>0 / \mathrm{B} 1>0$ and enhances with $\mathrm{A} 1<0 / \mathrm{B} 1<0$ and in the remaining region $(0.2,4.0)$ a reversed effect is noticed. The temperature enhances with $\mathrm{A} 1>0 / \mathrm{B} 1<0$ and reduces with $\mathrm{A} 1<0 / \mathrm{B} 1<0$ in the entire flow region (fig. $5 \mathrm{~b}$ ). From fig. $5 \mathrm{c}$ we find that in the flow regions $(0,0.5)$ and $(5,4.0)$ the nanoconcentration reduces with $\mathrm{A} 1>0$ and enhances with $\mathrm{A} 1<0$ while in the flow region $(0.5,2.5)$ the nanoconcentration enhances with $\mathrm{A} 1>0 / \mathrm{B} 1>0$, reduces with $\mathrm{A} 1<0 / \mathrm{B} 1<0$ (figs.6b\&6c).

The impact of $\mathrm{Nb}, \mathrm{Nt}$ qualitatively is same. The effect of the Brownian motion parameter $(\mathrm{Nb})$ and thermophoresis parameter $(\mathrm{Nt})$ is shown in Figs. $7 \mathrm{a} \& 8 \mathrm{a}$. The velocity decrease with $\mathrm{Nb}$ and enhances with $\mathrm{Nt}$ in the region $(0,1.0)$,in the region $(1,4)$ the velocity enhances with $\mathrm{Nb}$, reduces with $\mathrm{Nt} . \mathrm{An}$ increase in $\mathrm{Nb}$ leads to an enhancement in temperature in the entire flow region. It is observed that the effect of the thermophoresis parameter is remarkable on the temperature and concentration of the nanoparticles as expected. As the thermophoresis parameter (Nt) increases from 0.1 to 0.2 the temperature decreases and for higher $\mathrm{Nt} \geq 0.3$, it enhances in the flow region $(0,1.0)$ significantly throughout the region $(7 \mathrm{~b} \& 7 \mathrm{c})$. The nanoconcentration enhances with $\mathrm{N}$ bands reducers with $\mathrm{Nt}$ in the region $(0,1.0)$ and in the flow region(1.0,4.0), $\phi$ reduces with $\mathrm{Nb}$, reduces with $\mathrm{Nt}$.

Figs.9a-9c show the variation of velocity, temperature and nanoconcentration with chemical reaction parameter $(\gamma)$. The velocity reduces in the flow region $(0,1.5)$ and enhances in the region $(1.5,4.0)$ in both degenerating/ generation chemical reaction cases.The temperature increases with $0<\gamma>0$ in the entire flow region. An increase in $\gamma>0$ reduces the nanoconcentration in the entire flow region while an increase in $\gamma<0$, reduces $\theta$ and enhances $\phi$ in the region (1.0,4.0).

From 10a-10c represent the effect of Forcheimer parameter (fs) on the non-dimensional velocity, temperature and nanoparticle concentrtation. Fig.10a shows the variation of velocity with fs. In this case the velocity is found to depreciates in the flow region $(0,1.0)$ and enhances in the region $(1,4)$. 
From fig. $10 \mathrm{~b} \& 10 \mathrm{c}$, we find that as the Forcheimer parameter (fs) increases the temperature increases and nanoconcentration decreases in the flow region.Thus the inertia and boundary effects increases the temperature and depreciates the nanoparticle concentration. This is due to the thickening of the thermal boundary layer aand thinning of the solutal boundary layer as a result of increasing values of Forcheimer parameter (fs).

Figs.11a-11c represent $\mathrm{u}, \theta$ and $\phi$ with amplitude of the wavy surface ' $a$ '. From fig. 11a \& $11 \mathrm{~b}$, we find that an increase in amplitude enhances the velocity and temperature in the entire flow region. The nanoconcentration reduces in the region $(0,1.0)$ and enhance in $(1.0,4.0)$ with increase in ' $a$ ' .

Figs.12a-12d represent the variation of $\mathrm{u}, \mathrm{g}, \theta$ and $\phi$ with Lewis number Le. We find that an increase in Le increases the thickness of linear, angular velocities, thermal and nanoparticle volume fraction.

Figs.13a-13c depict the variation of $u \theta$ and $\phi$ with stream wise coordinate $(\xi)$. It can be seen from the profiles that an increase in stream wise coordinate decreases the velocity, temperature and increase nanoconcentration in the flow region $(0,1.0)$ and in the remaining region $(1,4)$, they experience a depreciation .

The skin freiction (Cf) at the wall is represented in table.2 for different variatioins. From the tabular values we find that the magnitude of the skin friction enhances with increase in N. Higher the thermal radiation $(\mathrm{Rd}) /$ dissipation $(\mathrm{Ec})$ smaller the skin friction at the wall. Higher the strength of the space/temperature dependent heat source larger magnitude of $\mathrm{Cf}$ and smaller $\mathrm{Cf}$ at the wall in these of heat sink, Cf reduces in both degenerating / generating chemical reaction cases. An increase in Forcheimer parameter (fs) / Lewis number (Le) leads to a decay in Cf at the wall, Also Cf grows with $\mathrm{Nt}$ and decays with $\mathrm{Nb}$ at the wall. An increase in amplitude of the wavy surface ' $a$ ' results in a depreciation in the skin friction at the wall.Also an increase in stream wise coordinate $(\xi)$ leads to a depreciation in the magnitude of $\mathrm{C}_{\mathrm{f}}$ at the wall.

The rate of heat transfer $(\mathrm{Nu})$ at the wall is displayed in table.2. From the tabular values we find that rate of heat transfer at the wall increases with increase in buoyancy parameter $(\mathrm{N})$. Higher the thermal radiation $(\mathrm{Rd})$ or dissipation smaller the Nusselt number. The rate of heat transfer increases at the wall with space/temperature dependent heat generating source $(\mathrm{A} 1>0, \mathrm{~B} 1>0)$ and reduces in the case of heat absorbing source $(\mathrm{A} 1<0, \mathrm{~B} 1<0)$. Higher Brownian motion/Forchheimer parameter leads to a depreciation in $\mathrm{Nu}$ at the wall.It enhances with increase in Nt.The rate of heat transfer at the wall decreases in both degenerating /generating chemical reaction cases.An increase in Le/amplitude (a) reduces the Nusselt number at the wall. The variation of $\mathrm{Nu}$ with stream wise coordinate $(\xi)$ shows that $\mathrm{Nu}$ increases with increase in enhances with ' $\xi$ '.

The rate of mass transfer (Sh) at the wall is displayed in table.2. From the tabular values we find that rate of mass transfer at the wall increases with increase in buoyancy ratio $(\mathrm{N})$. From the values we find that Sh decreases with increase in heat generating source and enhances with heat absorbing source. The Sherwood number reduces with increase in $\mathrm{Rd} /$ space dependent heat source at the wall.Higher the dissipation (Ec) smaller the Sherwood number at the wall. The rate of mass transfer at the wall reduces with $\mathrm{Nb}$ and enhances with $\mathrm{Nt} / \mathrm{fs}$.An increase in Le depreciates the rate of mass transfer at $\eta=0$. The rate of mass transfer at the wall enhances in both degenerating / generating chemical reaction cases. The variation of Sh with amplitude of the wavy surface $(a)$ and stream wise coordinate $(\xi)$ shows that Sh decreases with increase in ' $a$ ' and enhances with ' $\xi$ '.

\section{CONClusion}

The non-linear equations governing the flow, heat and mass transfer have been solved by RungeKutta-shooting technique.The findings of this analysis are

1] When the molecular buoyancy force dominates over the thermal buoyancy force the velocity enhances in $(0,1)$ and reduces when the buoyancy forces are in the same direction. The temperature enhances with increase in N.The nanoconcentration enhances in the region $(0,4.0)$ with increasing Nr.An increase in buoyancy ratio $(\mathrm{N})$ enhances the skin friction, Sherwood number and the Nusselt number on $\eta=0$.

2] Higher the thermal radiation ( $\mathrm{Rd})$ smaller the velocity in the region $(0,0.2)$ and larger in the region $(0.2,4.0)$. An increase in Rd leads to thickening of the thermal boundary layer,thickening of the solutal boundary layer in $(0,0.5)$, thinning of the solutal boundary layer in $(0.5,1.0)$ which results in an enhancement of the temperature and depreciation in the nano-concentration in the flow 
region.Higher the thermal radiation smaller the skin frtictiuon, the Nusselt and Sherwood numbers on the wall.

3] An increase in the strength of the space temperature dependent heat generating source $(\mathrm{A} 1>0$, B1>0)reduces the velocity,enhances temperature and nanoconcentration and while they experience reduction in the flow region with increasing strength of the absorbing heat source $(\mathrm{A} 1<0, \mathrm{~B} 1<0)$. Skin friction, Sherwood number and Sherwood number enhances, with $\mathrm{A} 1>0, \mathrm{~B} 1>0$ while they depreciates with $\mathrm{A} 1<0, \mathrm{~b} 1<0$ on $\eta=0$

4] The velocity reduces in the flow region $(0,1.5)$ and enhances in $(1.5,4.0)$ in both degenerating and generating chemical reaction cases. An increase in $\gamma$ enhances the temperature in the entire flow region. The nanoconcentration reduces in the flow $(0,1)$ in both degenerating / generating chemical reaction cases.In the flow region $(1,4)$ the concentration of the nanoparticles reduces with $\gamma>0$ and enhances with $\gamma<0$.The skin friction,Nusselt and Sherwood numbers reduce on $\eta=0$ in the degenerating generating chemical reaction cases .

5] The velocities decreases with thermophoresis parameter $(\mathrm{Nb})$ \& enhances Brownian motion parameter $(\mathrm{Nt})$ in $(0,1)$ and in $(1,4)$, $\mathrm{u}$ enhances with $\mathrm{Nb}$ and reduces with $\mathrm{Nt}$. The temperature and nanoconcentration enhances in the region $(0,1)$ and in the region $(1,4)$ the temperature enhances, the nanoconcentration reduces with $\mathrm{Nb}$ with Skin friction, $\mathrm{Nu}$, Sh enhances with $\mathrm{Nt}$ and reduces with $\mathrm{Nb}$ on the wall $\eta=0$.

6] Higher the dissipative force smaller the velocity, larger temperature and the nanoconcentration in the flow region. Skin friction, Nusselt number Sherwood number reduces with higher values of Ec on $\eta=0$.

7] An increase in fs reduces the velocityand nanoconcentration in $(0,1)$ and in the region $(1,4)$ velocity enhances,concentration reduces with fs. The temperature enhances in the entire flow region wirh fs.Thus the inertia and boundary effects increases the temperature and depreciates the nanoparticle concentration. This is due to the thickening of the thermal boundary layer aand thinning of the solutal boundary layer as a result of increasing values of Forcheimer parameter (fs). Skin friction, Nusselt number, Sherwood number reduces with higher values of fs on $\eta=0$.

8] An increased in Lewis number (Le) reduces the velocity, concentration depreciate with Le on $\eta=0$.

9] An increase in amplitude (a) enhances the velocity and temperature. The concentration reduces in the region $(0,1)$ and enhances in $(1,4)$ with increase in ' $a$ '. An increase in amplitude $(a)$ deprectates Nuselt and Sherwood numbers and enhances the skin friction on the wall $\eta=0$.

10] An increase in stream wise coordinate $(\xi)$ decreases the velocity, temperature while enhances the concentration in the flow region $(0,1)$ and depreciates in $(1,4)$. An increase in stream wise coordinate $(\xi)$ reduces skin friction and enhances Nusselt and Sherwood numbers on $\eta=0$.
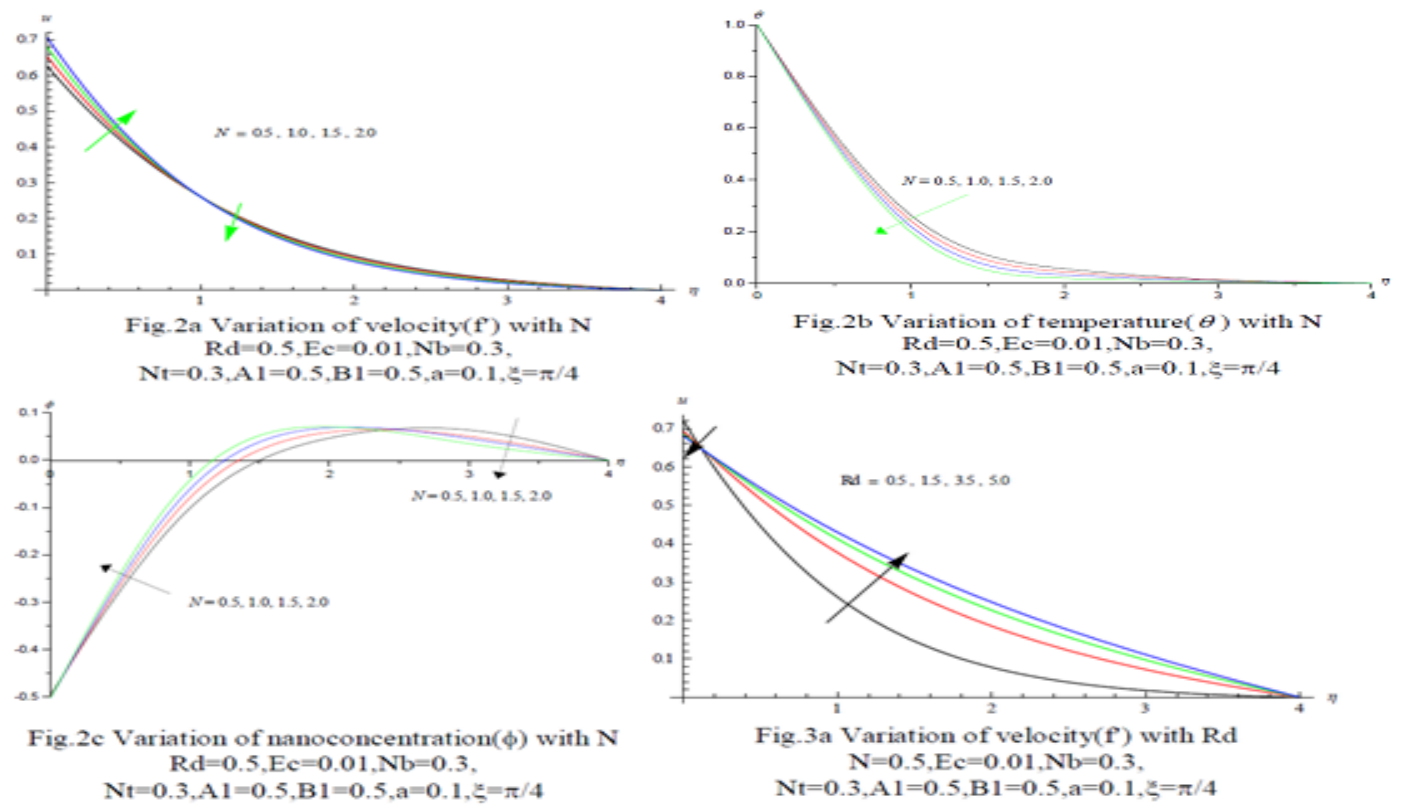


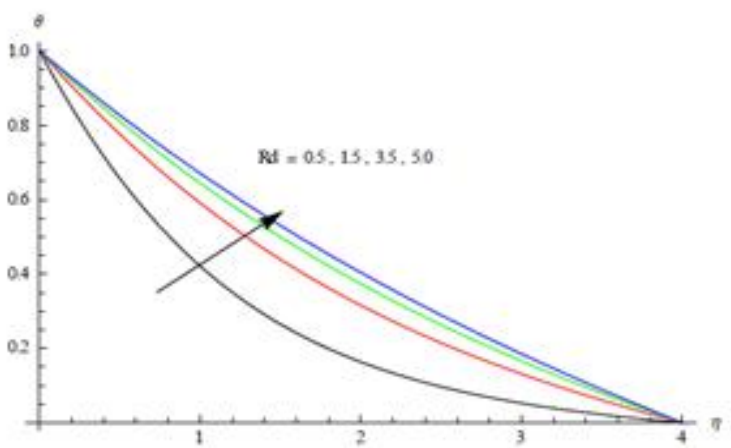

Fig.3b Variation of temperature $(\theta)$ with Rd $\mathrm{N}=0.5, \mathrm{Ec}=0.01, \mathrm{Nb}=0.3$,

$\mathrm{N} t=0.3, \mathrm{~A} 1=0.5, \mathrm{~B} 1=0.5, \mathrm{a}=0.1, \xi=\pi / 4$

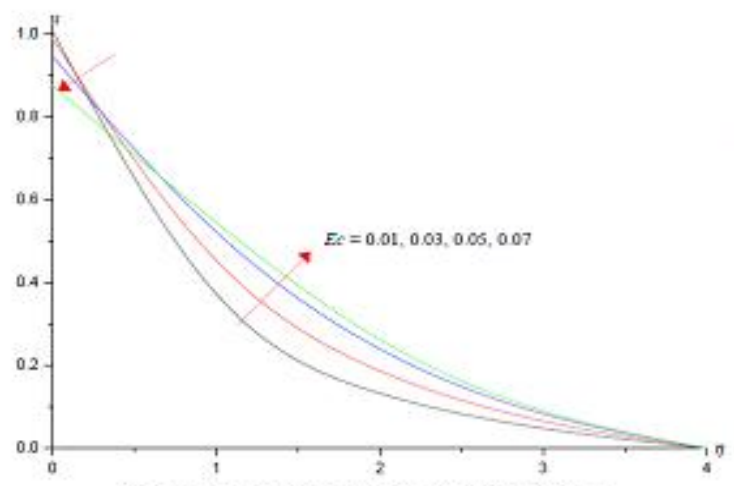

Fig.4a Variation of velocity(f) with Ec $\mathrm{N}=0.5, \mathrm{Rd}=0.5, \mathrm{Nb}=0.3$,

$\mathrm{Nt}=0.3, \mathrm{Al}=0.5, \mathrm{Bl}=0.5, \mathrm{a}=0.1, g=\pi / 4$

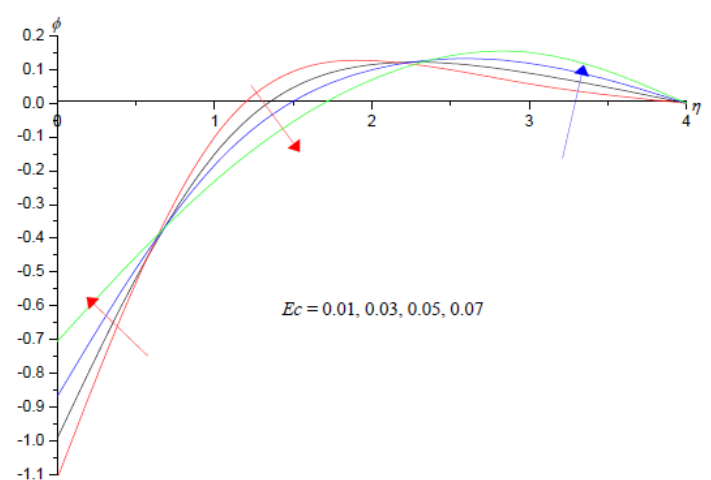

Fig. $4 \mathrm{c}$ Variation of nanoconcentration $(\phi)$ with Ec $\mathrm{N}=0.5, \mathrm{Rd}=0.5, \mathrm{Nb}=0.3, \mathrm{Nt}=0.3$,

$\mathrm{Al}=0.5, \mathrm{~B} 1=0.5, \mathrm{a}=0.1, \xi=\pi / 4, \mathrm{Le}=2$

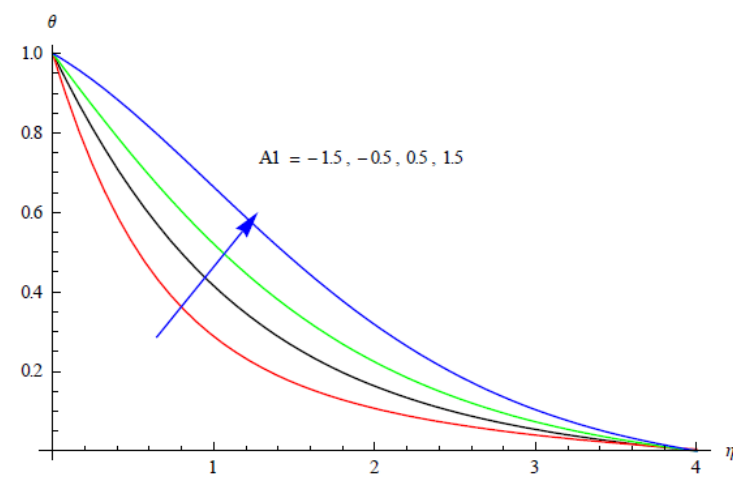

Fig. $5 \mathrm{~b}$ Variation of temperature $(\theta)$ with $\mathrm{A} 1$ $\mathrm{N}=0.5, \mathrm{Rd}=0.5, \mathrm{Ec}=0.01, \mathrm{Nb}=0.3$, $\mathrm{Nt}=0.3, \mathrm{~B} 1=0.5, \mathrm{a}=0.1, \xi=\pi / 4$

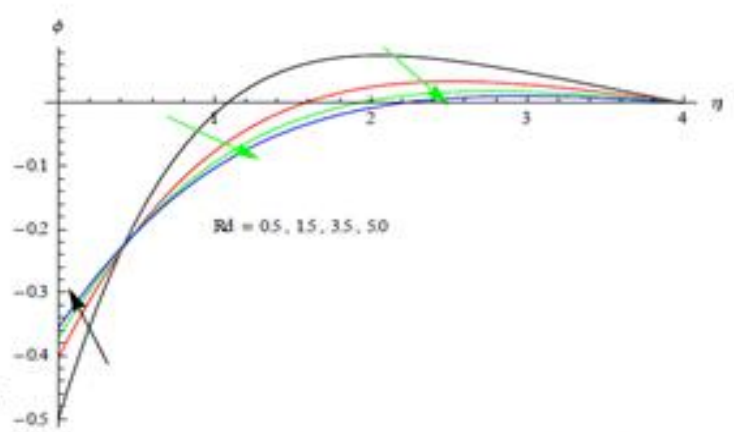

Fig.3c Variation of nanoconcentration $(\phi)$ with $\mathrm{Rd}$ $\mathrm{N}=0.5, \mathrm{Ec}=0.01, \mathrm{Nb}=0.3$,

$\mathrm{Nt}=0.3, \mathrm{Al}=0.5, \mathrm{~B} 1=0.5, \mathrm{a}=0.1, \xi=\pi / 4$

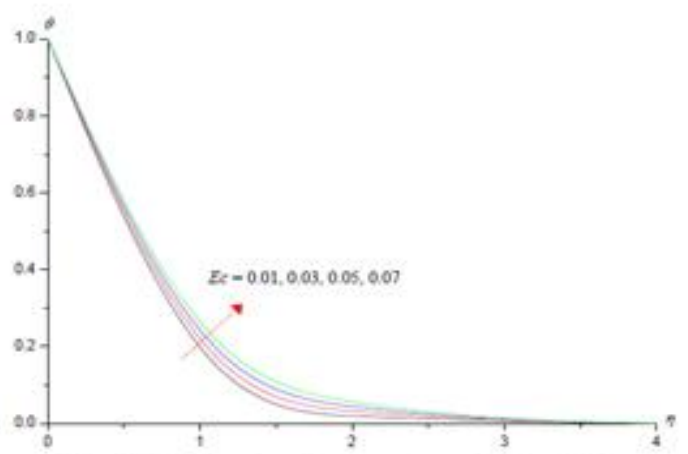

Fig. $4 \mathrm{~b}$ Variation of temperature $(\theta)$ with $\mathrm{Ec}$ $\mathrm{N}=0.5, \mathrm{Rd}=0.5, \mathrm{Nb}=0.3, \mathrm{Nt}=0.3$. $\mathrm{Al}=0.5, \mathrm{Bl}=0.5, \mathrm{a}=0.1, \xi=\pi / 4, \mathrm{Le}=2$

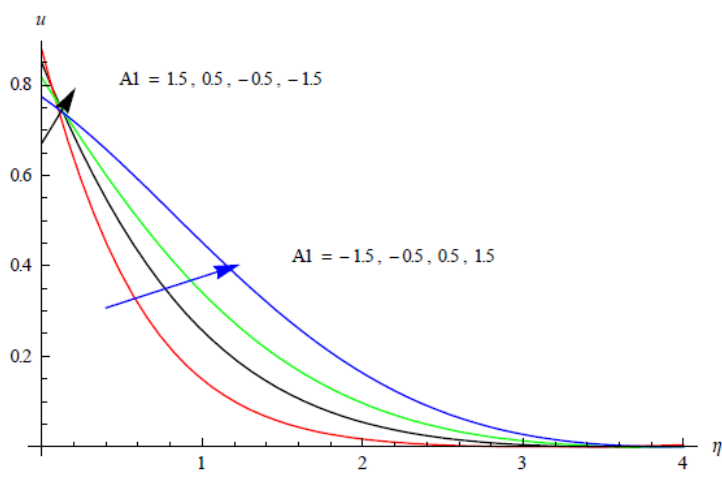

Fig.5a Variation of velocity $\left(\mathrm{f}^{\prime}\right)$ with $\mathrm{A} 1$ $\mathrm{N}=0.5, \mathrm{Rd}=0.5, \mathrm{Ec}=0.01, \mathrm{Nb}=0.3$, $\mathrm{Nt}=0.3, \mathrm{~B} 1=0.5, \mathrm{a}=0.1, \xi=\pi / 4$

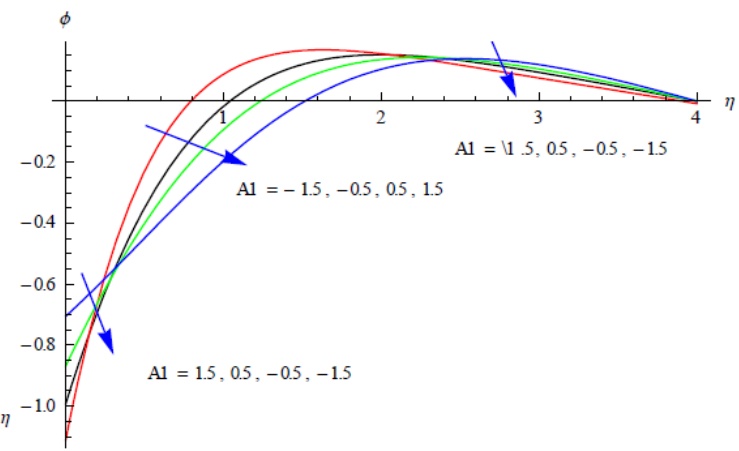

Fig. $5 \mathrm{c}$ Variation of nanoconcentration $(\phi)$ with $\mathrm{A} 1$ $\mathrm{N}=0.5, \mathrm{Rd}=0.5, \mathrm{Ec}=0.01, \mathrm{Nb}=0.3$, $\mathrm{Nt}=0.3, \mathrm{~B} 1=0.5, \mathrm{a}=0.1, \xi=\pi / 4$ 


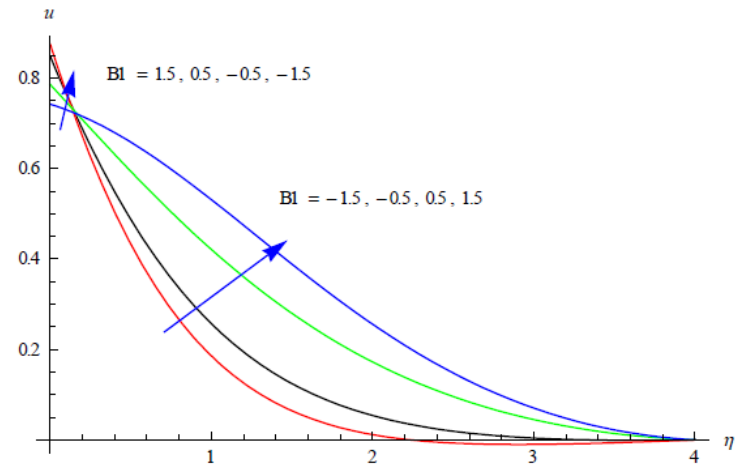

Fig.6a Variation of velocity $\left(\mathrm{f}^{\prime}\right)$ with $\mathrm{B} 1$ $\mathrm{N}=0.5, \mathrm{Rd}=0.5, \mathrm{Ec}=0.01, \mathrm{Nb}=0.3$, $\mathrm{Nt}=0.3, \mathrm{~A} 1=0.5, \mathrm{a}=0.1, \xi=\pi / 4$

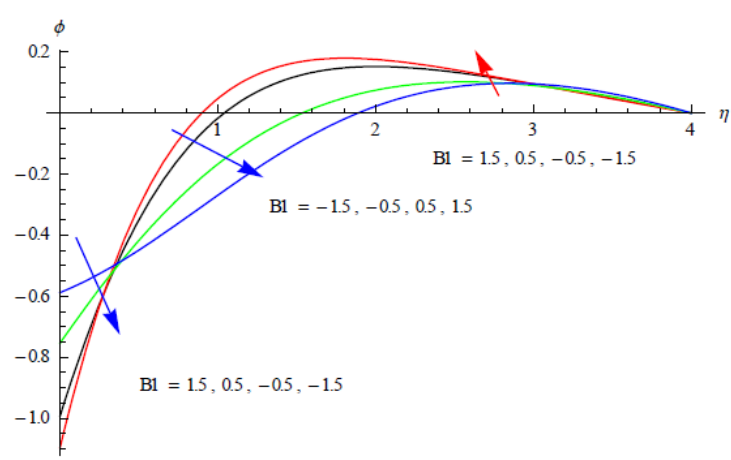

Fig.6c Variation of nanoconcentration $(\phi)$ with B1 $\mathrm{N}=0.5, \mathrm{Rd}=0.5, \mathrm{Ec}=0.01, \mathrm{Nb}=0.3$, $\mathrm{Nt}=0.3, \mathrm{~A} 1=0.5, \mathrm{a}=0.1, \xi=\pi / 4$

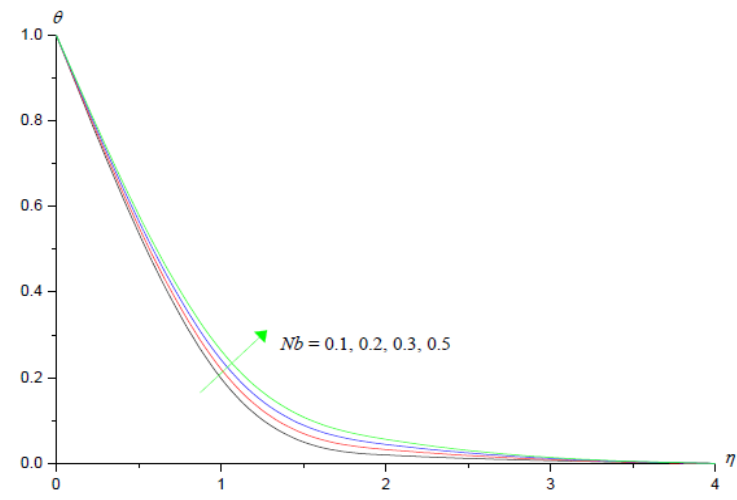

Fig. $7 \mathrm{~b}$ Variation of temperature $(\theta)$ with $\mathrm{Nb}$ $\mathrm{N}=0.5, \mathrm{Rd}=0.5, \mathrm{Ec}=0.01, \mathrm{Nt}=0.3, \mathrm{~A} 1=0.5$,

$$
\mathrm{B} 1=0.5, \mathrm{a}=0.1, \xi=\pi / 4
$$

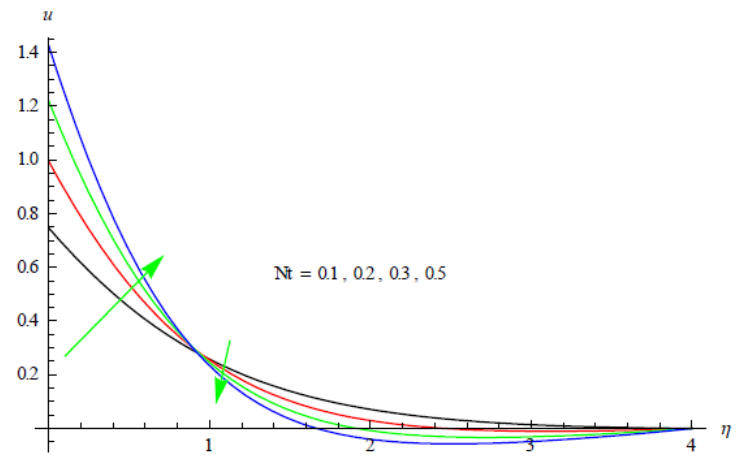

Fig. 8a Variation of velocity $\left(\mathrm{f}^{\prime}\right)$ with $\mathrm{Nt}$ $\mathrm{N}=0.5, \mathrm{Rd}=0.5, \mathrm{Ec}=0.01, \mathrm{Nb}=0.3$, $\mathrm{A} 1=0.5, \mathrm{~B} 1=0.5, \mathrm{a}=0.1, \xi=\pi / 4$

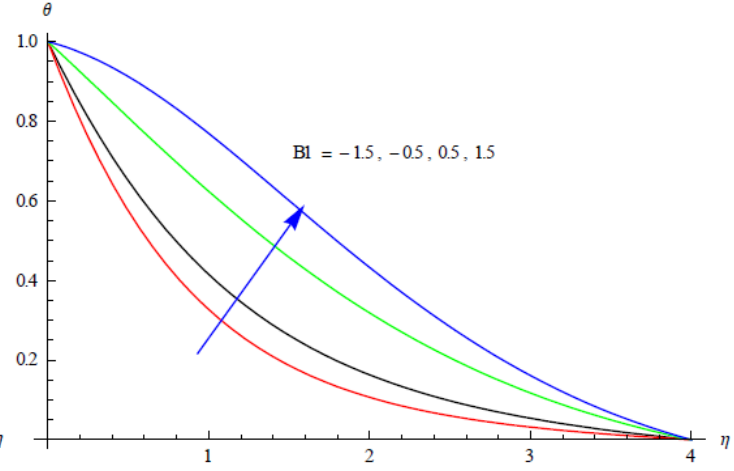

Fig.6b Variation of temperature $(\theta)$ with $\mathrm{B} 1$ $\mathrm{N}=0.5, \mathrm{Rd}=0.5, \mathrm{Ec}=0.01, \mathrm{Nb}=0.3$, $\mathrm{Nt}=0.3, \mathrm{~A} 1=0.5, \mathrm{a}=0.1, \xi=\pi / 4$

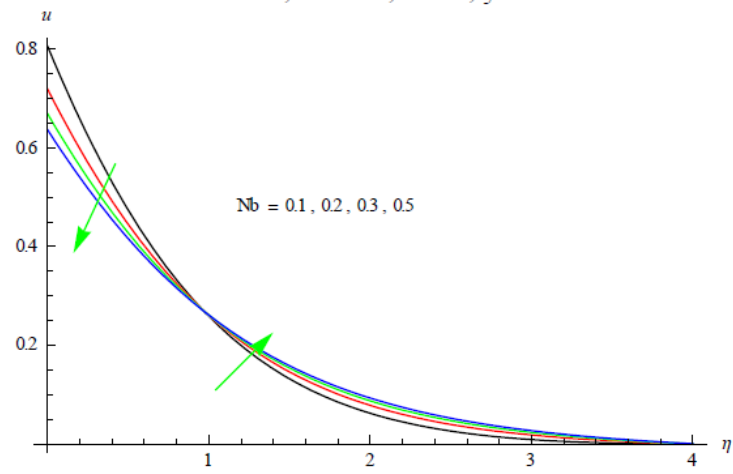

Fig.7a Variation of velocity $\left(\mathrm{f}^{\prime}\right)$ with $\mathrm{Nb}$ $\mathrm{N}=0.5, \mathrm{Rd}=0.5, \mathrm{Ec}=0.01$,

$\mathrm{Nt}=0.3, \mathrm{~A} 1=0.5, \mathrm{~B} 1=0.5, \mathrm{a}=0.1, \xi=\pi / 4$

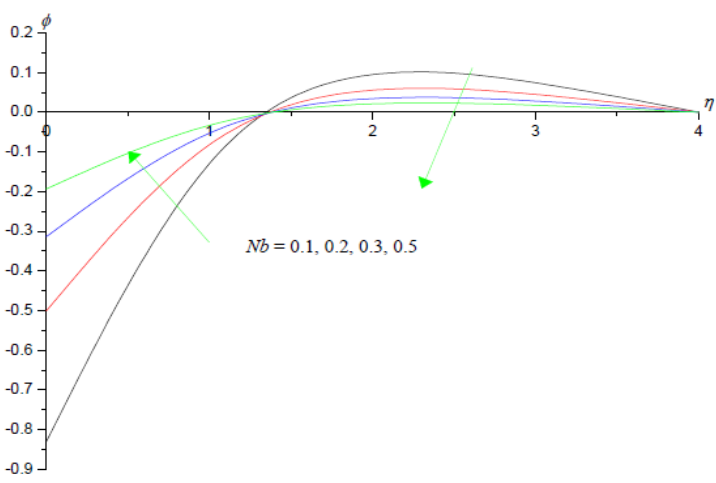

Fig. $7 \mathrm{c}$ Variation of nanoconcentration $(\phi)$ with $\mathrm{Nb}$ $\mathrm{N}=0.5, \mathrm{Rd}=0.5, \mathrm{Ec}=0.01, \mathrm{Nt}=0.3$, $\mathrm{A} 1=0.5, \mathrm{~B} 1=0.5, \mathrm{a}=0.1, \xi=\pi / 4$

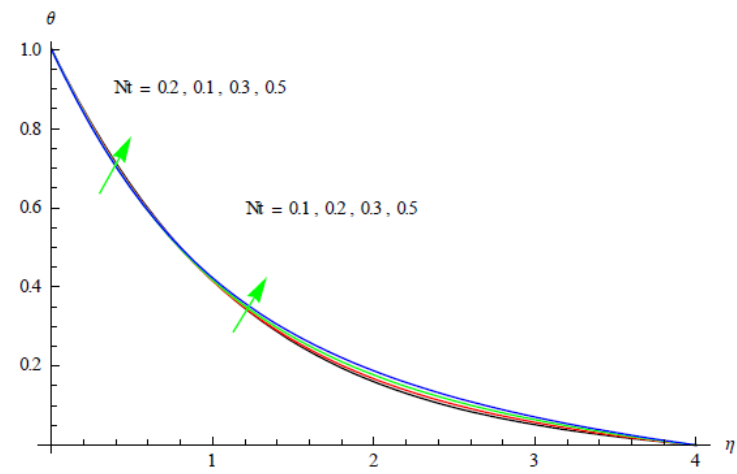

Fig. $8 \mathrm{~b}$ Variation of temperature $(\theta)$ with $\mathrm{Nt}$ $\mathrm{N}=0.5, \mathrm{Rd}=0.5, \mathrm{Ec}=0.01, \mathrm{Nb}=0.3$, $\mathrm{A} 1=0.5, \mathrm{~B} 1=0.5, \mathrm{a}=0.1, \xi=\pi / 4$ 
Convective Heat and Mass Transfer Flow of Nanofluid past a Vertical Wavy Wall with Thermal Radiation, Chemical Reaction with Space and Temperature Dependent Heat Sources

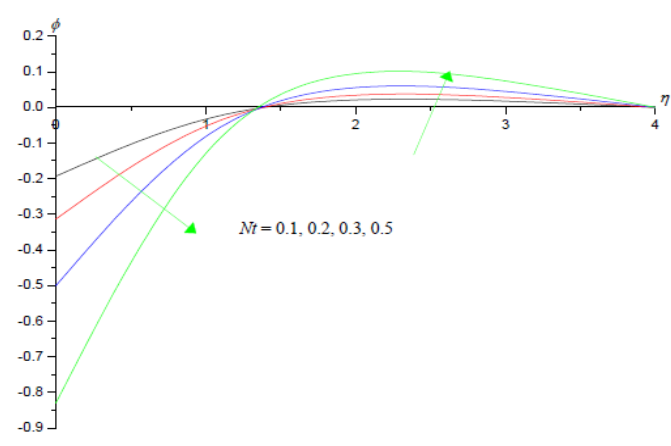

Fig. $8 \mathrm{c}$ Variation of nanoconcentration $(\phi)$ with $\mathrm{Nt}$ $\mathrm{N}=0.5, \mathrm{Rd}=0.5, \mathrm{Ec}=0.01, \mathrm{Nb}=0.3$, $\mathrm{A} 1=0.5, \mathrm{~B} 1=0.5, \mathrm{a}=0.1, \xi=\pi / 4$

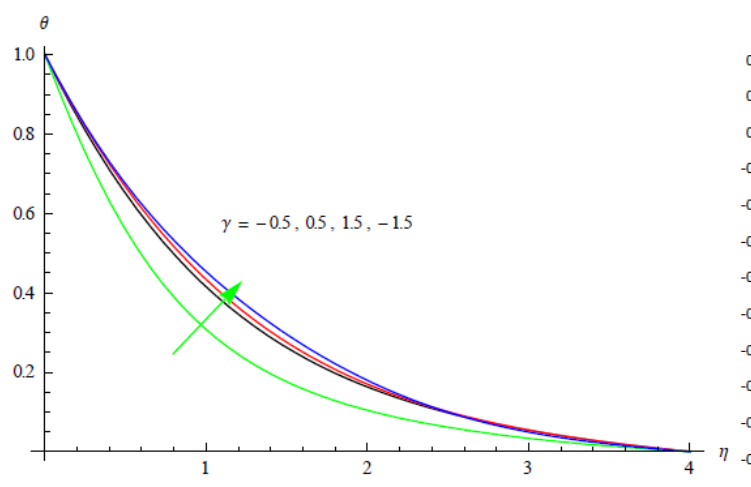

Fig.9b Variation of temperature $(\theta)$ with $\gamma$ $\mathrm{N}=0.5, \mathrm{Rd}=0.5, \mathrm{Ec}=0.01, \mathrm{Nb}=0.3$, $\mathrm{Nt}=0.3, \mathrm{~A} 1=0.5, \mathrm{~B} 1=0.5, \mathrm{a}=0.1, \xi=\pi / 4$

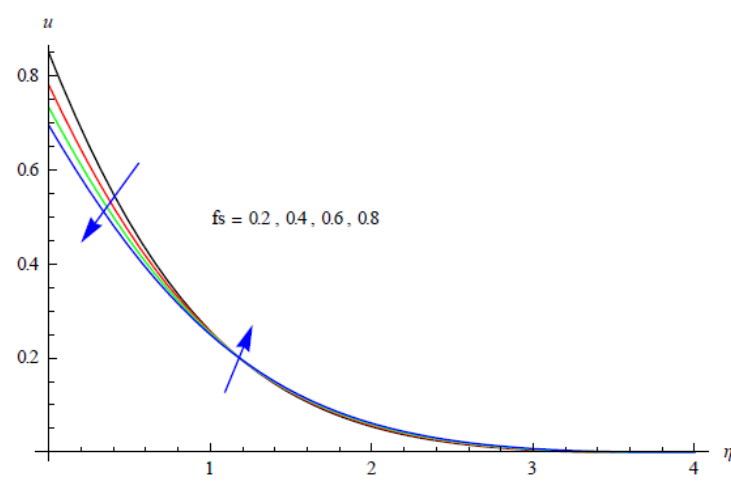

Fig. 10a Variation of velocity $\left(\mathrm{f}^{\prime}\right)$ with $\mathrm{fs}$ $\mathrm{N}=0.5, \mathrm{Rd}=0.5, \mathrm{Ec}=0.01, \mathrm{Nb}=0.3$, $\mathrm{Nt}=0.3, \mathrm{~A} 1=0.5, \mathrm{~B} 1=0.5, \mathrm{a}=0.1, \xi=\pi / 4$

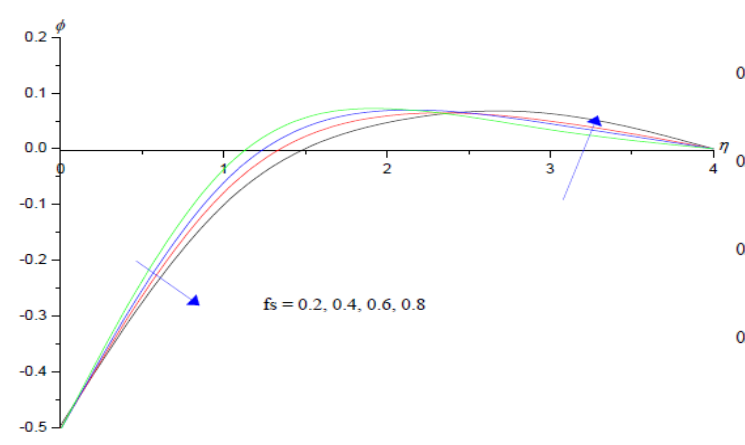

Fig. 10c Variation of nanoconcentration $(\phi)$ with fs $\mathrm{N}=0.5, \mathrm{Rd}=0.5, \mathrm{Ec}=0.01, \mathrm{Nb}=0.3$, $\mathrm{Nt}=0.3, \mathrm{~A} 1=0.5, \mathrm{~B} 1=0.5, \mathrm{a}=0.1, \xi=\pi / 4$

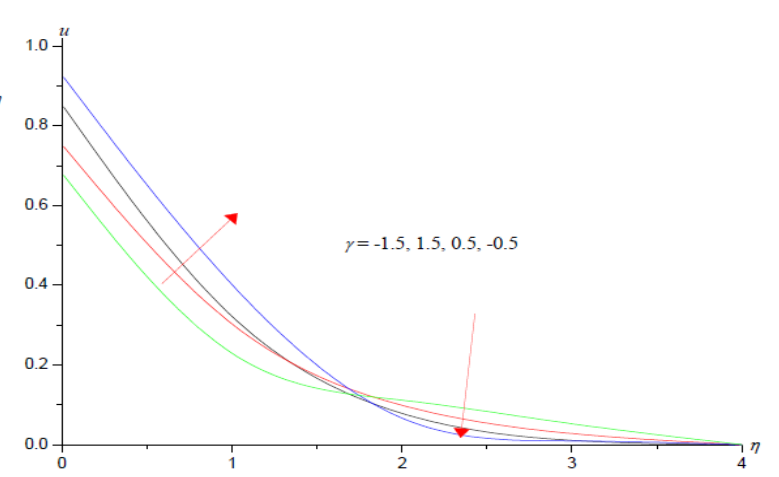

Fig.9a Variation of velocity $\left(f^{\prime}\right)$ with $\gamma$ $\mathrm{N}=0.5, \mathrm{Rd}=0.5, \mathrm{Ec}=0.01, \mathrm{Nb}=0.3$,

$\mathrm{Nt}=0.3, \mathrm{~A} 1=0.5, \mathrm{~B} 1=0.5, \mathrm{a}=0.1, \xi=\pi / 4$

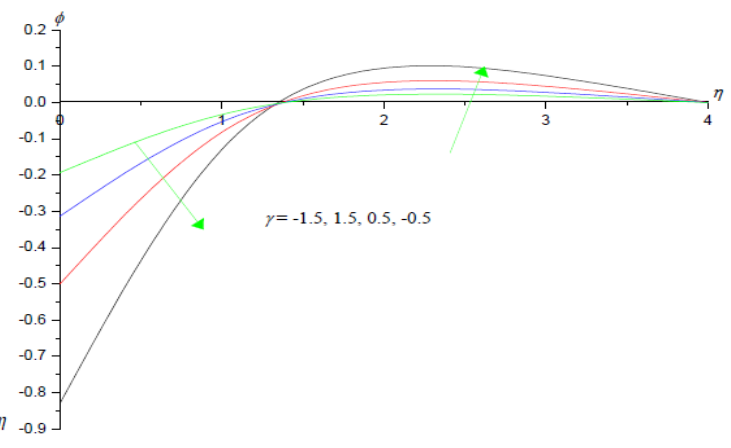

Fig.9c Variation of nanoconcentration $(\phi)$ with $\gamma$ $\mathrm{N}=0.5, \mathrm{Rd}=0.5, \mathrm{Ec}=0.01, \mathrm{Nb}=0.3$,

$\mathrm{Nt}=0.3, \mathrm{~A} 1=0.5, \mathrm{~B} 1=0.5, \mathrm{a}=0.1, \xi=\pi / 4$

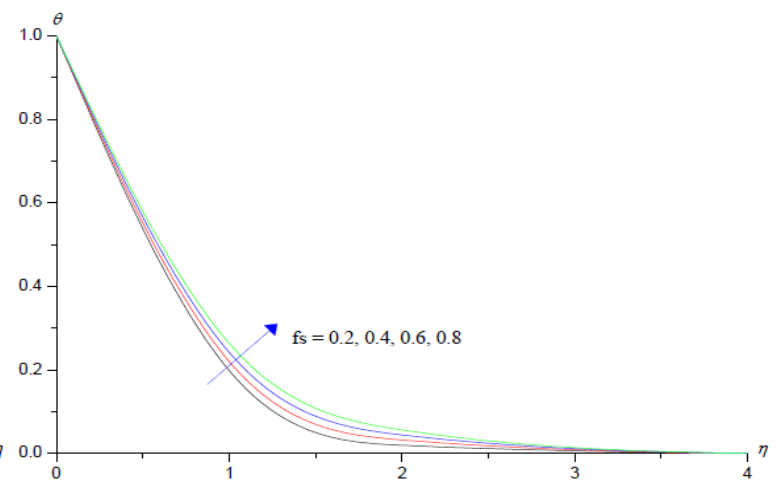

Fig. 10b Variation of temperature $(\theta)$ with fs $\mathrm{N}=0.5, \mathrm{Rd}=0.5, \mathrm{Ec}=0.01, \mathrm{Nb}=0.3$, $\mathrm{Nt}=0.3, \mathrm{~A} 1=0.5, \mathrm{~B} 1=0.5, \mathrm{a}=0.1, \xi=\pi / 4$

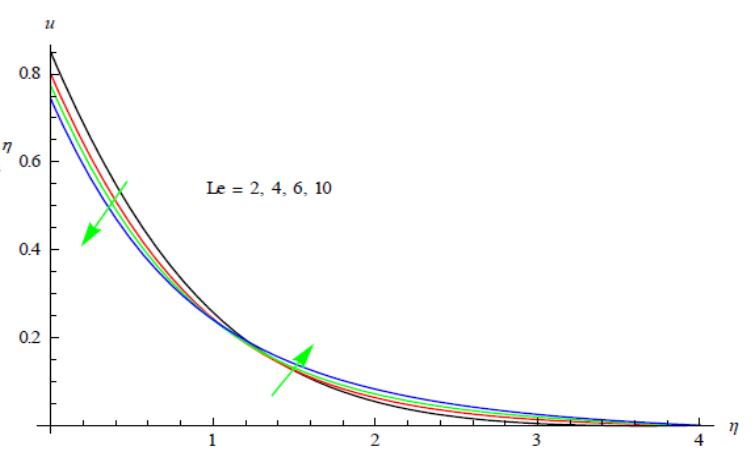

Fig. 11a Variation of velocity $\left(\mathrm{f}^{\prime}\right)$ with Le $\mathrm{N}=0.5, \mathrm{Rd}=0.5, \mathrm{Ec}=0.01, \mathrm{Nb}=0.3$, $\mathrm{Nt}=0.3, \mathrm{~A} 1=0.5, \mathrm{~B} 1=0.5, \mathrm{a}=0.1, \xi=\pi / 4$ 
Convective Heat and Mass Transfer Flow of Nanofluid past a Vertical Wavy Wall with Thermal Radiation, Chemical Reaction with Space and Temperature Dependent Heat Sources

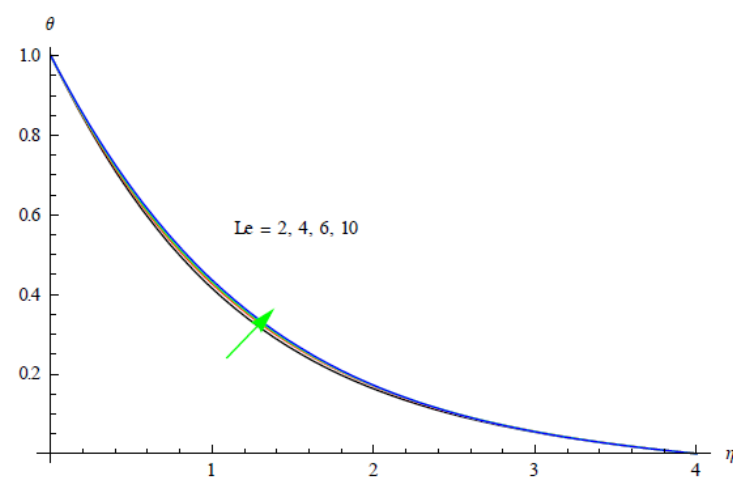

Fig.11b Variation of temperature $(\theta)$ with Le $\mathrm{N}=0.5, \mathrm{Rd}=0.5, \mathrm{Ec}=0.01, \mathrm{Nb}=0.3$, $\mathrm{Nt}=0.3, \mathrm{~A} 1=0.5, \mathrm{~B} 1=0.5, \mathrm{a}=0.1, \xi=\pi / 4$

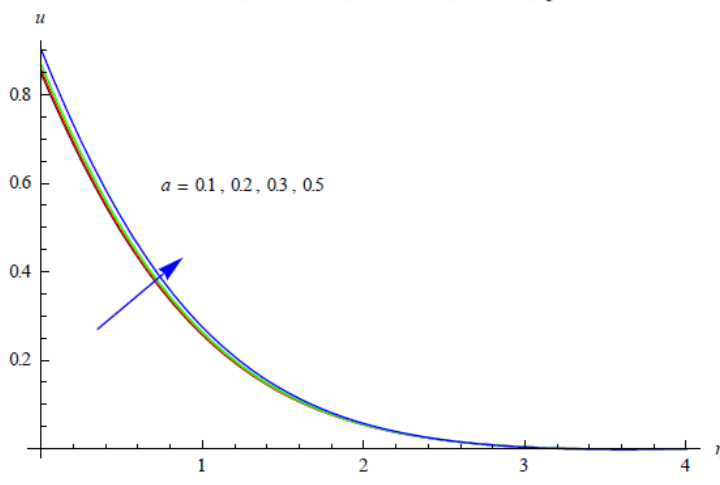

Fig.12a Variation of velocity $\left(\mathrm{f}^{\prime}\right)$ with a $\mathrm{N}=0.5, \mathrm{Rd}=0.5, \mathrm{Ec}=0.01, \mathrm{Nb}=0.3$,

$\mathrm{Nt}=0.3, \mathrm{~A} 1=0.5, \mathrm{~B} 1=0.5, \mathrm{a}=0.1, \xi=\pi / 4$

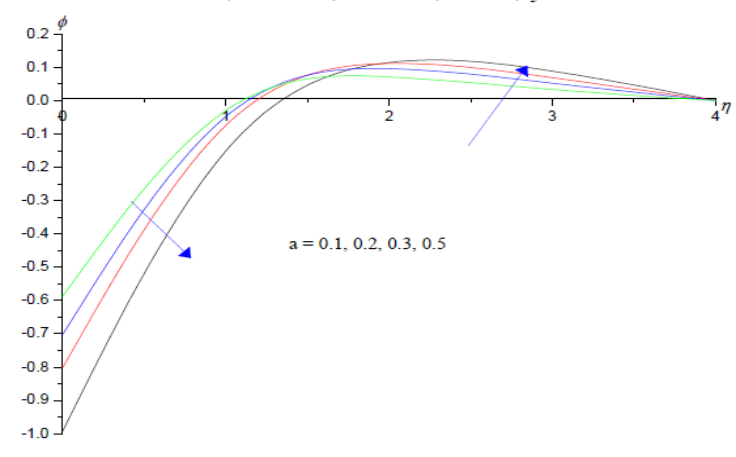

Fig. 12c Variation of nanoconcentration $(\phi)$ with a $\mathrm{N}=0.5, \mathrm{Rd}=0.5, \mathrm{Ec}=0.01, \mathrm{Nb}=0.3$, $\mathrm{Nt}=0.3, \mathrm{~A} 1=0.5, \mathrm{~B} 1=0.5, \mathrm{a}=0.1, \xi=\pi / 4$

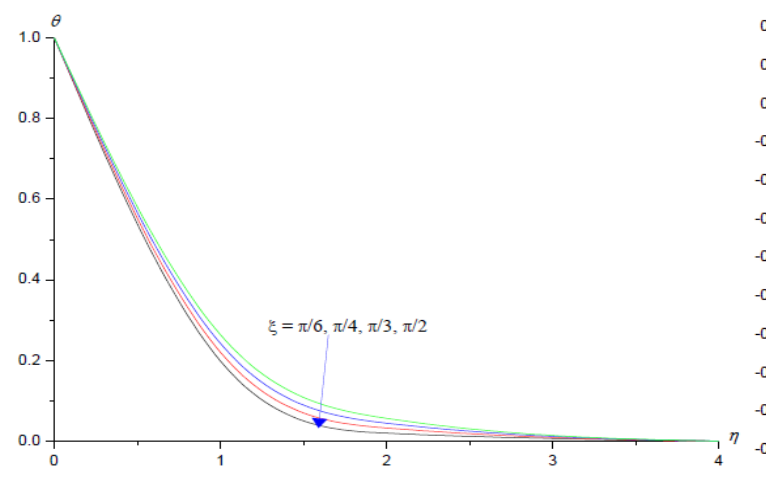

Fig.13b Variation of temperature $(\theta)$ with $\xi$ $\mathrm{N}=0.5, \mathrm{Rd}=0.5, \mathrm{Ec}=0.01, \mathrm{Nb}=0.3$, $\mathrm{Nt}=0.3, \mathrm{~A} 1=0.5, \mathrm{~B} 1=0.5, \mathrm{a}=0.1$

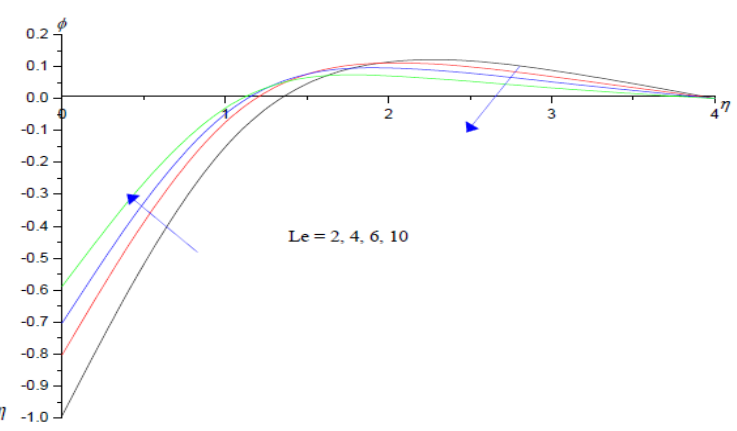

Fig.11c Variation of nanoconcentration $(\phi)$ with Le $, \mathrm{N}=0.5, \mathrm{Rd}=0.5, \mathrm{Ec}=0.01, \mathrm{Nb}=0.3$, $\mathrm{Nt}=0.3, \mathrm{~A} 1=0.5, \mathrm{~B} 1=0.5, \mathrm{a}=0.1, \xi=\pi / 4$

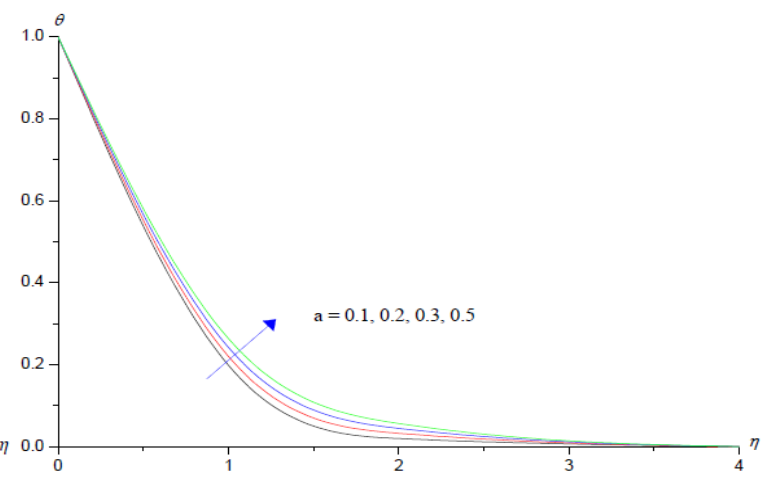

Fig. 12b Variation of temperature $(\theta)$ with a $\mathrm{N}=0.5, \mathrm{Rd}=0.5, \mathrm{Ec}=0.01, \mathrm{Nb}=0.3$, $\mathrm{Nt}=0.3, \mathrm{~A} 1=0.5, \mathrm{~B} 1=0.5, \mathrm{a}=0.1, \xi=\pi / 4$

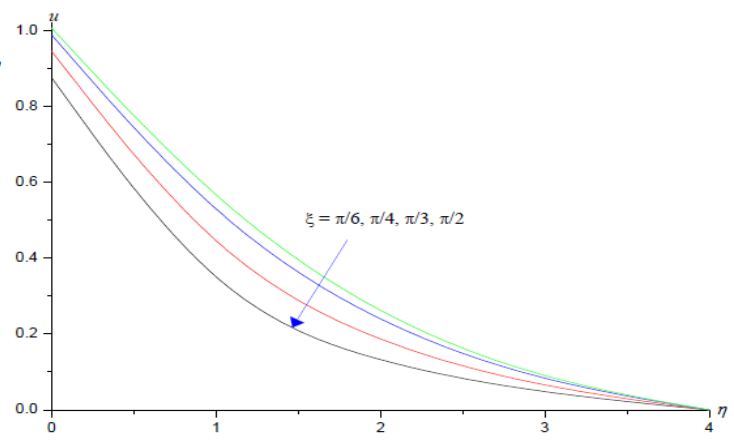

Fig.13a Variation of velocity $\left(\mathrm{f}^{\prime}\right)$ with $\xi$ $\mathrm{N}=0.5, \mathrm{Rd}=0.5, \mathrm{Ec}=0.01, \mathrm{Nb}=0.3$, $\mathrm{Nt}=0.3, \mathrm{~A} 1=0.5, \mathrm{~B} 1=0.5, \mathrm{a}=0.1, \xi=\pi / 4$

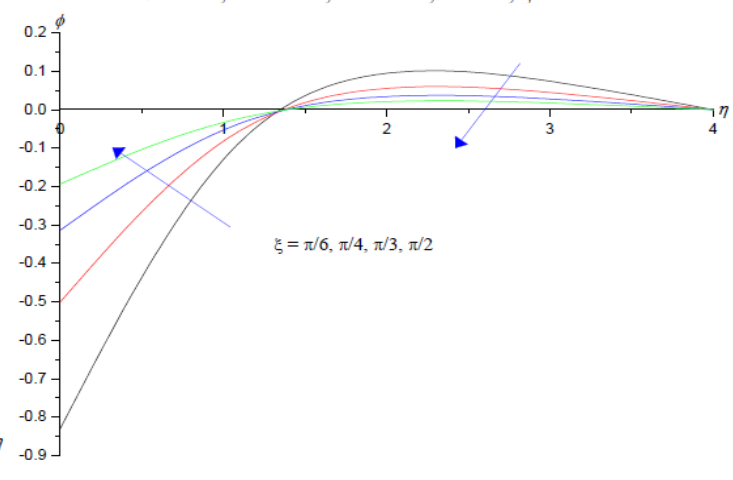

Fig. $13 \mathrm{c}$ Variation of nanoconcentration $(\phi)$ with $\xi$ $\mathrm{N}=0.5, \mathrm{Rd}=0.5, \mathrm{Ec}=0.01, \mathrm{Nb}=0.3$, $\mathrm{Nt}=0.3, \mathrm{~A} 1=0.5, \mathrm{~B} 1=0.5, \mathrm{a}=0.1$ 
Convective Heat and Mass Transfer Flow of Nanofluid past a Vertical Wavy Wall with Thermal Radiation, Chemical Reaction with Space and Temperature Dependent Heat Sources

Table2. Skin Friction $\left(\tau_{x}\right)$, Nusslet number $(\mathrm{Nu})$ and Sherwood Number $(\mathrm{Sh})$ at $\eta=0$

\begin{tabular}{|c|c|c|c|c|c|c|c|c|c|}
\hline \multicolumn{2}{|c|}{ Parameter } & $\tau \mathbf{x}(\mathbf{0})$ & $\mathrm{Nu}(\mathbf{0})$ & $\operatorname{Sh}(0)$ & \multicolumn{2}{|c|}{ Parameter } & $\tau \mathbf{X}(\mathbf{0})$ & $\mathrm{Nu}(\mathbf{0})$ & $\operatorname{Sh}(\mathbf{0})$ \\
\hline \multirow[t]{4}{*}{$\mathrm{N}$} & 0.5 & -0.494305 & -0.494305 & -0.792374 & \multirow[t]{4}{*}{ B1 } & 0.5 & -0.861994 & 0.832395 & -1.66479 \\
\hline & 1 & -0.537661 & -0.537661 & -0.797519 & & 1.5 & -1.1163 & 1.08385 & -2.1677 \\
\hline & 1.5 & -0.581011 & -0.581011 & -0.80254 & & -0.5 & -0.39144 & 0.373203 & -0.746407 \\
\hline & 2 & -0.624345 & -0.624345 & -0.80744 & & -1.5 & -0.110211 & 0.104128 & -0.208256 \\
\hline \multirow[t]{4}{*}{$\mathrm{Rd}$} & 0.5 & -0.646003 & 0.809846 & -0.809846 & \multirow[t]{4}{*}{$\gamma$} & 0.5 & -0.861994 & 0.832395 & -1.66479 \\
\hline & 1.5 & -0.392428 & 0.48927 & -0.48927 & & 1.5 & -0.831937 & 0.787047 & -1.57409 \\
\hline & 3.5 & -0.324659 & 0.404067 & -0.404067 & & -0.5 & -1.02049 & 1.08202 & -2.16404 \\
\hline & 5 & -0.292584 & 0.363828 & -0.363828 & & -1.5 & -0.825406 & 0.780076 & -1.56015 \\
\hline \multirow[t]{4}{*}{ Ec } & 0.01 & -0.861994 & 0.832395 & -1.66479 & \multirow[t]{4}{*}{ fs } & 0.2 & -0.861994 & 0.832395 & -1.66479 \\
\hline & 0.03 & -0.861241 & 0.83166 & -1.66332 & & 0.4 & -0.729958 & 0.818463 & -1.63693 \\
\hline & 0.05 & -0.860524 & 0.830959 & -1.66192 & & 0.6 & -0.64856 & 0.8081 & -1.6162 \\
\hline & 0.07 & -0.859806 & 0.830257 & -1.66051 & & 0.8 & -0.587976 & 0.799267 & -1.59853 \\
\hline \multirow[t]{4}{*}{$\mathrm{Nb}$} & 0.1 & -0.790142 & 0.825171 & -1.37529 & \multirow[t]{4}{*}{$\mathrm{a}$} & 0.1 & -0.861994 & 0.832395 & -1.66479 \\
\hline & 0.2 & -0.646003 & 0.809846 & -0.809846 & & 0.2 & -0.869177 & 0.829568 & -1.65914 \\
\hline & 0.3 & -0.564756 & 0.800672 & -0.50042 & & 0.3 & -0.87935 & 0.825361 & -1.65072 \\
\hline & 0.5 & -0.512648 & 0.794566 & -0.305602 & & 0.5 & -0.907049 & 0.81296 & -1.62592 \\
\hline \multirow[t]{4}{*}{$\mathrm{Nt}$} & 0.2 & -0.694279 & 0.820572 & -0.984686 & \multirow[t]{4}{*}{$\xi$} & $\pi / 6$ & -0.86308 & 0.831978 & -1.66396 \\
\hline & 0.4 & -1.10769 & 0.847242 & -2.71118 & & $\pi / 4$ & -0.861994 & 0.832395 & -1.66479 \\
\hline & 0.6 & -1.50101 & 0.866015 & -4.50328 & & $\pi / 3$ & -0.860893 & 0.832813 & -1.66563 \\
\hline & 0.8 & -1.87472 & 0.879401 & -6.33169 & & $\pi / 2$ & -0.859773 & 0.833231 & -1.66646 \\
\hline \multirow[t]{4}{*}{ A1 } & 0.5 & -0.861994 & 0.832395 & -1.66479 & & & & & \\
\hline & 1.5 & -1.37235 & 1.34164 & -2.64923 & & & & & \\
\hline & -0.5 & -0.566758 & 0.543703 & -1.08741 & & & & & \\
\hline & -1.5 & -0.247629 & 0.235471 & -0.470942 & & & & & \\
\hline
\end{tabular}

\section{REFERENCES}

[1] Abo-Eldahab E. M. Abd El Aziz M. Hall and ion-slip effect on MHD free convective heat generating flow past a semi-infinite vertical flat plate Phs. Scripta, v.61,p344, (2000).

[2] Abo-Eldahab E. M. Salem A. M. Hall effects on MHD free convection flow of a non- Newtonian power-law fluid at a stretching surface Int. Commun. Heat Mass, v.31(3), pp.343- 354, (2004)

[3] Ali F. M. Nazar R. Arifin N. M. and Pop I. Effect of Hall current on MHD mixed convection boundary layer flow over a stretched vertical flat plate Meccanica, v.46, pp.1103-1112, (2011).

[4] Ching-Yang Cheng : Soret and Dufour effects on free convection boundary layers over an inclined wavy surface in a porous medium. Int. Comm. Heat and Mass transfer, v.38, pp.1050-1055, (2011).

[5] Hady F.M. Mohamed R.A. Mahdy A. : MHD free convective flow along a vertical wavy surface with heat generation or absorption effect Int. Comm. Heat Mass transfer, v.33, (2006).

[6] HossainM.A KabirS and ReesD.A.S:Z.Angew.Math.phys.V.53pp.48-52(2002)

[7] Mallikarjuna B : Convective heat and mass transfer in viscous fluid flow over a porous medium Ph.D. thesis JNTUA Ananthapuramu June 2014.

[8] Mohamed Abd El-Aziz Effects of Hall current on the flow and heat transfer of a nanofluid over a stretching sheet with partial slip International Journal of Modern Physics C Vol. 24 No. 7 (2013) 1350044.

[9] Ramakrishna G.N., and Satyanarayana K : "Effect of heat sources and thermal radiation on convective heat transfer flow past a vertical wavy wall with variable viscosity and thermal conductivity", International Journal of Mathematical Archieve (IJMA), Vol.10, No.11, pp:15-25, (2019). ISSN 22295046, web: www.ijma.info.

[10] Ranganadha Reddy, P : Hydromagnetic Convective Heat and Mass Transfer Flow in Viscous Fluid with Variable Viscosity and Thermal Conductivity, Ph.D. Thesis, Yogivemana University, Kadapa, A.P., India (2020).

[11] Rees D.A.S Pop I : Free convection induced by a vertical wavy surface with uniform heat flux in a porous medium. ASME J. Heat Transfer V.117 pp. 547-550 (1995).

[12] ReesD.A.S and PopI:ASME Journal of Heat TransferV.116pp.505-508(1994)

[13] Sulochana C and Sandeep Naramgari : MHD flow over a permeable stretching/shrinking sheet on a nanofluid in porous medium with suction/injection, International Conference on Research in Education and Science (ICRES), Antalya, Turkey, pp.273-285, April 23-26, (2015)

[14] Vendabai K : Mathematical modelling of heat transfer in nanofluids, Ph.D. Thesis, Sri Padmavathi Mahila Visvavidyalayam, (2015). 
Convective Heat and Mass Transfer Flow of Nanofluid past a Vertical Wavy Wall with Thermal Radiation, Chemical Reaction with Space and Temperature Dependent Heat Sources

[15] Xiaohonh Su Liancun Zheng Hall effect on MHD flow and heat transfer of nanofluids over a stretching wedge in the presence of velocity slip and Joule heating Cent. Eur. J. Phys. 11(12), pp.1694 - 1703, (2013).

\section{AUTHOR'S BIOGRAPHY}

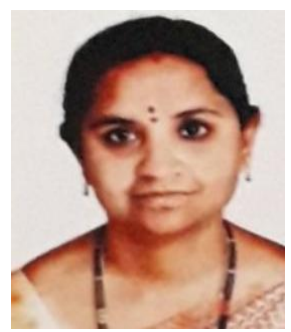

Dr. M .Nagasasikala, is a Lecturer in Mathematics, Incharge of the department of Mathematics, Government Degree College, Autonomous, Anantapur, A.P, India since 2008. She did her M.Sc in 2001 from Sri Krishnadevaraya University, Anantapur, A.P., India. She completed her Ph.D from Sri Padmavathi Mahila University, Tirupathi on the topic Convective heat and mass Transfer Flow of a Viscous Fluid through a Porous medium in Channels/Ducts in 2016. She has published 8 papers in different journals.

Citation: Dr. M. Nagasasikala., Convective Heat and Mass Transfer Flow of Nanofluid past a Vertical Wavy Wall with Thermal Radiation, Chemical Reaction with Space and Temperature Dependent Heat Sources, International Journal of Scientific and Innovative Mathematical Research (IJSIMR), vol. 8, no. 9, pp. 22-35, 2020. Available : DOI: https://doi.org/10.20431/2347-3142.0809004

Copyright: (C) 2020 Authors. This is an open-access article distributed under the terms of the Creative Commons Attribution License, which permits unrestricted use, distribution, and reproduction in any medium, provided the original author and source are credited. 